

\title{
Mechanism of chirality reversal for planar interface domain walls in exchange-coupled hard/soft magnetic bilayers
}

J Mccord, Y Henry, Thomas Hauet, F Montaigne, Eric E. Fullerton, S Mangin

\section{- To cite this version:}

J Mccord, Y Henry, Thomas Hauet, F Montaigne, Eric E. Fullerton, et al.. Mechanism of chirality reversal for planar interface domain walls in exchange-coupled hard/soft magnetic bilayers. Physical Review B: Condensed Matter and Materials Physics (1998-2015), 2008, 10.1103/PhysRevB.78.094417 . hal-01345263

\section{HAL Id: hal-01345263 \\ https://hal.science/hal-01345263}

Submitted on 13 Jul 2016

HAL is a multi-disciplinary open access archive for the deposit and dissemination of scientific research documents, whether they are published or not. The documents may come from teaching and research institutions in France or abroad, or from public or private research centers.
L'archive ouverte pluridisciplinaire HAL, est destinée au dépôt et à la diffusion de documents scientifiques de niveau recherche, publiés ou non, émanant des établissements d'enseignement et de recherche français ou étrangers, des laboratoires publics ou privés. 


\title{
Mechanism of chirality reversal for planar interface domain walls in exchange-coupled hard/soft magnetic bilayers
}

\author{
J. McCord, ${ }^{1}$ Y. Henry, ${ }^{2}$ T. Hauet, ${ }^{3}$ F. Montaigne, ${ }^{3}$ Eric E. Fullerton, ${ }^{4}$ and S. Mangin ${ }^{3, *}$ \\ ${ }_{1}^{1}$ Institute for Metallic Materials, IFW Dresden, PF 270116, D-01171 Dresden, Germany \\ ${ }^{2}$ IPCMS, UMR CNRS 7504, Université Louis Pasteur, F-67034 Strasbourg Cedex 2, France \\ ${ }^{3}$ LPM, Nancy-Université, UMR CNRS 7556, F-54506 Vandoeuvre Cedex, France \\ ${ }^{4}$ Center for Magnetic Recording Research, University of California, San Diego, La Jolla, California 92093-0401, USA
}

(Received 1 July 2008; published 17 September 2008)

\begin{abstract}
The mechanism of chirality reversal for a planar interface domain wall in a hard/soft magnetic bilayer has been identified by combining magnetoresistance measurements, modeling, and direct magnetic domain observations. The reversal occurs through IDW nucleation and lateral domain wall propagation. Over an unpredicted wide range of applied magnetic fields, the chirality transition takes place by an unwinding followed by a rewinding of the IDW. The chirality transition mechanism of phase transition could be identified from a micromagnetic analysis of the lateral magnetic domain wall orientation. Up to three magnetization phases coexist in the uniaxial material during reversal.
\end{abstract}

DOI: 10.1103/PhysRevB.78.094417

PACS number(s): 75.60.Ch, 71.70.Gm, 75.10.Pq, 75.60.Jk

\section{INTRODUCTION}

The control of nonuniform magnetic orders which occur at surfaces, ${ }^{1}$ domain walls in thin films ${ }^{2}$ and strips, ${ }^{3}$ and magnetic vortices in disks and rings ${ }^{4,5}$ is one of the key issues in the optimization of magnetic nanostructures. In particular, chiral magnetic orders such as vortices ${ }^{6}$ are appealing as they could be used to store binary information and experiments have been devoted to the identification of the direction of magnetization curling in such structures. Recently, the possibility of a controlled switching of the vortex core polarization, i.e., the vortex chirality, has been demonstrated. ${ }^{7}$

Prototypical chiral magnetic orders are also found in thinfilm exchange-spring magnets. ${ }^{8,9}$ These structures are composed of exchange-coupled hard and soft magnetic layers. The hard magnetic phase provides the pinning which stabilizes the magnetization of the soft magnetic phase in a particular orientation via the exchange coupling across the interface. Upon application of an external magnetic field, the soft layer magnetization rotates to line up with the field while being pinned at the interface. This results in a rotation angle that increases with increasing distance from the hard material. A spiral magnetic order called interface domain wall (IDW) thus forms which disappears upon removal of the field as the soft layer magnetization rotates back in alignment with the hard phase. Such planar walls are thought to play an important role in the exchange-bias properties of antiferromagnetic/ferromagnetic bilayers. ${ }^{10-12}$

Works on $\mathrm{SmCo} / \mathrm{Fe}$ exchange-spring films using torque magnetometry $^{13}$ and magneto-optical indicator film technique, ${ }^{14}$ as well as on $\mathrm{TbFe} / \mathrm{GdFe}$ bilayers, ${ }^{15,16}$ have shown that an interface domain wall can undergo an abrupt inversion of its chirality when it is submitted to a continuously rotating magnetic field (not sufficient to affect significantly the hard magnetic phase). As the field is rotated away from the pinning direction, the soft layer first adopts a spiral spin configuration $\sigma^{+}$where the magnetization rotates in the same direction as the field as one moves away from the hard/ soft interface. The situation is sketched in Fig. 1(a) for a clockwise $(\mathrm{CW})$ rotation of the field $H_{\text {rot }}$. The $\sigma^{+}$configuration remains the ground state for field angles up to $180^{\circ}$ with respect to the hard layer magnetization (assuming a ferromagnetic interface coupling). However, as the angle exceeds $180^{\circ}$, this configuration becomes metastable and, at a critical value $\psi_{c}$, the soft layer magnetization switches irreversibly to another spiral configuration $\sigma^{-}$of lower energy and opposite chirality as shown in Fig. 1(b).

The evolution of $\psi_{c}$ with the applied field magnitude proved to be quite complex. Platt et al. ${ }^{13}$ found a nonmonotonic dependence of $\psi_{c}$ and modeled the chirality switch in the $\mathrm{SmCo} / \mathrm{Fe}$ system by in-plane unwinding at low field and out-of-plane fanning at high field. For the hard/soft $\mathrm{TbFe} /$ $\mathrm{GdFe}$ system, apart from the two chirality states $\sigma^{+}$and $\sigma^{-}$, another stable configuration labeled $\sigma^{0}$ was identified, in which the GdFe moments are trapped along the direction of

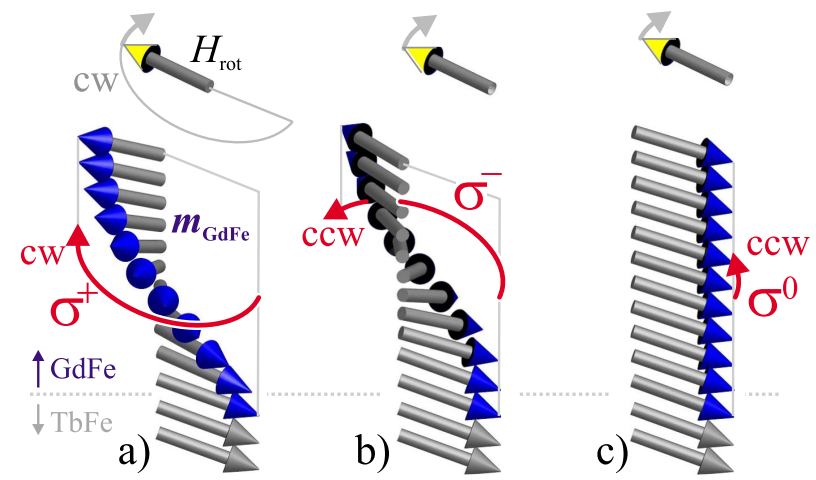

FIG. 1. (Color online) Possible states of magnetization in exchange-spring hard/soft $\mathrm{TbFe} / \mathrm{GdFe}$ samples. (a) Spiral spin configuration $\sigma^{+}$where the magnetization $m_{\mathrm{GdFe}}$ rotates along the sense of field rotation. (b) Twisted soft layer magnetization $\sigma^{-}$with spiral configuration of opposite chirality. (c) Spin state $\sigma^{0}$ in which the $\mathrm{GdFe}$ moments are aligned along the direction of interfacial pinning (see text for details). A clockwise (CW) rotating magnetic field $H_{\text {rot }}$ is assumed in the sketches. The spin states $\sigma^{+}, \sigma^{-}$, and $\sigma^{0}$ are labeled in (a)-(c). The senses of rotation of $m_{\mathrm{GdFe}}, \mathrm{CW}$ or counterclockwise $(\mathrm{CCW})$, are indicated. 
interfacial pinning [Fig. 1(c)]. It arises from the combined effects of the interface exchange interaction and a uniaxial anisotropy in the plane of the film which stabilizes the $\sigma^{0}$ state during the unwinding process. Thus, for moderate fields, transitions from $\sigma^{+}$to $\sigma^{0}$, and then to $\sigma^{-}$, were found. ${ }^{16}$ The equilibrium magnetic configurations $\sigma^{+}, \sigma^{-}$, and $\sigma^{0}$ are reproduced quantitatively by a spin chain model, containing no free parameter. However, the prediction of irreversible transitions between the magnetic configurations is out of the scope of this model. The moderate field regime is not even expected from $1 D$ micromagnetic simulations since not only is the calculated energy of the $\sigma^{-}$configuration lower than that of $\sigma^{0}$, but the height of the energy barrier between $\sigma^{+}$and $\sigma^{0}$ is larger than the height of the energy barrier between $\sigma^{+}$and $\sigma^{-}$.

\section{EXPERIMENT AND RESULTS}

To shed light on the micromagnetic mechanism of chirality reversal, we combined low-temperature anisotropic magnetoresistance (AMR) measurements and direct magnetic domain observations by magneto-optical Kerr microscopy ${ }^{6,17}$ in an optical cryostat. The complementary experiments were carried out at $20 \mathrm{~K}$, under very similar conditions, on the same amorphous hard/soft $\mathrm{Tb}_{45} \mathrm{Fe}_{55}(50 \mathrm{~nm}) /$ $\mathrm{Gd}_{40} \mathrm{Fe}_{60}(50 \mathrm{~nm})$ ferrimagnetic bilayer sample. Details on sample preparation are given in Ref. 15. Prior to measurements, the latter was cooled down from room temperature in a large magnetic field of several kOe applied along the easy axis of the soft GdFe layer. This field cooling procedure induced in the hard $\mathrm{TbFe}$ layer a high remanent magnetization homogeneously fixed along the easy direction. In the following, the orientations of the domain walls at the various transitions are analyzed and the mechanism of chirality transition is deduced.

Figure 2 displays AMR measurements and results from the $1 D$ model for a clockwise rotating magnetic field of $H_{\text {rot }}=220$ Oe, together with magnetic domain observations. For the experiments the rotation field velocity was about 20 $\mathrm{deg} / \mathrm{min}$. An almost perfect agreement between the model and the AMR data is obtained over a wide range of field angles. By comparing the results of the simulations with the resistivity data [Fig. 2(a)] it is found that the IDW starts to wind in the direction of the magnetic field rotation (clockwise winding, $\sigma^{+}$state), then unwinds to form a $\sigma^{0}$ configuration and finally rewinds to the counterclockwise twisted $\sigma^{-}$ state. ${ }^{16}$ As already mentioned, the transition angle cannot be predicted from our micromagnetic approach. Kerr microscopy images have been taken at various field angles [Figs. 2(b) $-2(\mathrm{~m})$ ] during these transitions. The magneto-optical signal is obtained from the topmost $20-30 \mathrm{~nm}$ of the GdFe film. Both subsequently occurring chirality transitions, from $\sigma^{+}$ $\rightarrow \sigma^{0}$ and then from $\sigma^{0} \rightarrow \sigma^{-}$, are clearly identified. Note that both magnetization processes occur in a narrower angle range as compared to the AMR data. This discrepancy is likely due to the difference in the surface areas probed by the two techniques $\left(200 \times 200 \mu \mathrm{m}^{2}\right.$ for Kerr imaging and 5 $\times 10 \mathrm{~mm}^{2}$ for AMR). As illustrated in Figs. 2(c) $-2(\mathrm{~g})$, the $\sigma^{+} \rightarrow \sigma^{0}$ transition is dominated by lateral domain nucleation,
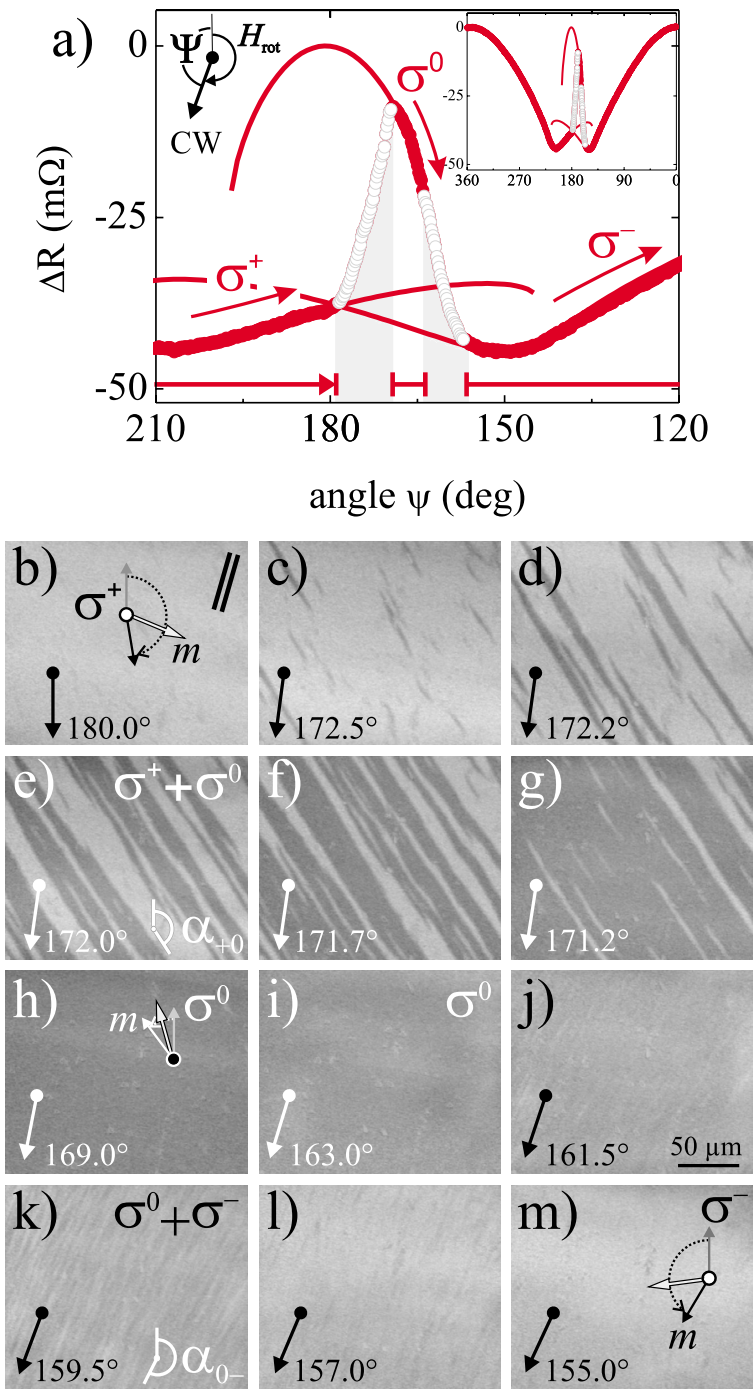

FIG. 2. (Color online) (a) Change in sample resistance (symbols $)$ for a clockwise field rotation $\left(H_{\text {rot }}=220\right.$ Oe $)$ together with results from the spin chain model (lines). The solid symbols indicate the reversible parts of the AMR curve, where an agreement with the computation results is found. The irreversible switching regimes are labeled by open symbols. (b)-(m) Magnetic domain images during the chirality transition process of (a). The soft layer easy axis of magnetization is vertical and the interface pinning direction points upward. $\alpha^{+0}$ and $\alpha^{0-}$ define the angle between the easy axis and the $\sigma^{+} / \sigma^{0}$ and $\sigma^{0} / \sigma^{-}$domain walls, respectively. The rotating field angle $\psi$ and the magneto-optical sensitivity direction (II) are indicated. The lateral resolution for all images is below $1 \mu \mathrm{m}$.

followed by lateral domain wall (LDW) motion. No domain activity occurs in the angle range where only the $\sigma^{0}$ state is observed [Figs. 2(h) and 2(i)]. The $\sigma^{0} \rightarrow \sigma^{-}$transition takes place in a similar way to the $\sigma^{+} \rightarrow \sigma^{0}$ one [Figs. 2(j)-2(1)]. However, the domain density during reversal is much higher. The reduced domain contrast is a direct consequence of the alignment of the magnetization relative to the magnetooptical sensitivity direction. The formation of the ripple-like domains is attributed to the fact that the $\sigma^{0}$ and $\sigma^{-}$states have similar energies at the critical angle, ${ }^{16}$ which facilitates 



FIG. 3. (Color online) (a) Change in sample resistance (symbols) and modeling results (lines) for a rotating field of $H_{\text {rot }}$ $=230$ Oe. (b)-(e) Evolution of the magnetic domain structure during the chirality transition process displayed in (a). The inset of (c) shows a schematic representation of the zigzag $\sigma^{0} / \sigma^{-}$walls formed as the $\sigma^{-}$phase expands inside the $\sigma^{0}$ domains.

the nucleation of many domains. Significantly, the LDWs are aligned along particular orientation angles, $\alpha^{+0}$ and $\alpha^{0-}$, respectively (see Fig. 2). As we will further discuss later, these are determined by the demand for zero net magnetic charge on the LDW.

The magnetization processes for a slightly larger rotating field of $H_{\text {rot }}=230 \mathrm{Oe}$ are displayed in Fig. 3. From the resistivity measurements, the development of the $\sigma^{+}$configuration can still be clearly identified. However, unlike before, in the process of switching from the $\sigma^{+}$state to the $\sigma^{-}$state, the sample resistance never reaches the high value characteristic of a laterally uniform $\sigma^{0}$ state. Domain observations reveal the mechanism of reversal. As in the lower field example, domains of $\sigma^{0}$ configuration nucleate and grow by LDW propagation [Fig. 3(b)]. Note the equivalence in domain wall (DW) orientation angle $\alpha^{+0}$ by comparing Figs. 2(c)-2(g) and Figs. 3(b) and 3(c). Yet, as seen in Fig. 3(c), a third phase rapidly nucleates inside the $\sigma^{0}$ domains. From the magneto-optical contrast and more importantly the LDW orientation angle, close to $\alpha^{0-}$ in Figs. 2(j)-2(1), this third phase
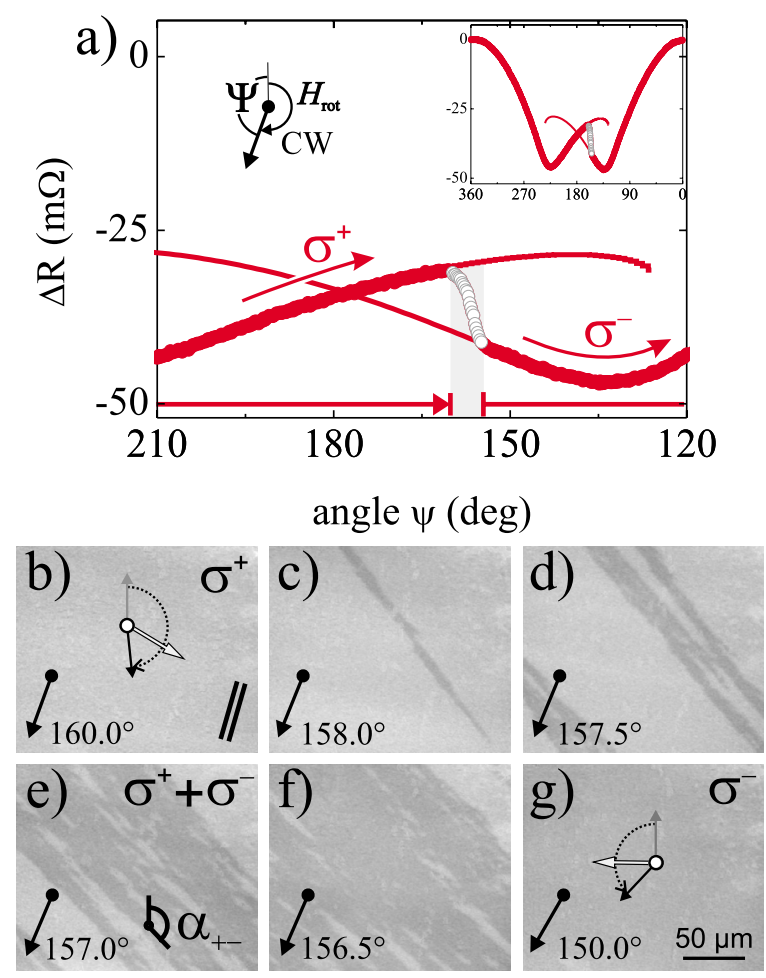

FIG. 4. (Color online) (a) Change in sample resistance and modeling results for $H_{\text {rot }}=265$ Oe. (b) $-(\mathrm{g})$ Corresponding magnetic domain evolution around the critical field angle $\psi_{c}$.

is identified as being in the $\sigma^{-}$state. This demonstrates the possible coexistence of all three phases $\sigma^{+}, \sigma^{0}$, and $\sigma^{-}$with a remarkable organization: The $\sigma^{-}$domains are surrounded by the intermediary $\sigma^{0}$ phase, which is contained by the original $\sigma^{+}$phase. As the field is rotated further, the chirality change proceeds by a two-step $\sigma^{+} / \sigma^{0}$ - and $\sigma^{0} / \sigma^{-}$-DW motion process, which eventually leads to the predicted $\sigma^{-}$state. In the course of this process, zigzag $\sigma^{0} / \sigma^{-}$DWs are formed next to the $\sigma^{+} / \sigma^{0}$ DWs [see inset of Fig. 3(c)]. With the identification of the simultaneous occurrence of three chirality phases, we will now focus on the $\sigma^{+}$to $\sigma^{-}$transition at large field.

Resistivity measurements at $H_{\text {rot }}=265 \mathrm{Oe}$, as shown in Fig. 4(a), suggest a direct $\sigma^{+}$to $\sigma^{-}$chirality conversion, as expected from the $1 D$ model. Domain imaging [Figs. 4(b) $-4(\mathrm{~g})]$ reveals a seemingly single step process at the critical angle $\psi_{c} \sim 157^{\circ}$, in agreement with the AMR data. As in the previous two cases, the chirality transition is dominated by LDW motion. However, a few dissimilarities in the processes are noticeable from the Kerr microscopy images. First of all, the domain boundaries are more irregular in shape than in the former cases. Also, the magnetization distribution inside the already switched $\sigma^{-}$domains displays some inhomogeneities. Importantly, with the average magnetization of the $\sigma^{-}$phase being oriented very differently from that of the $\sigma^{0}$ phase [see sketches in Fig. 2(a)], a strong modification of the LDW orientation is expected in the event of a direct $\sigma^{+}$to $\sigma^{-}$transition. Yet, no such alteration of the LDW angle is found as one moves from the intermediate field regime to the high field one [compare Figs. 2(c)-2(g) and Figs. 4(c)-4(f)].

For clarification, the LDW orientation angles expected for the various possible transitions were modeled. They were 


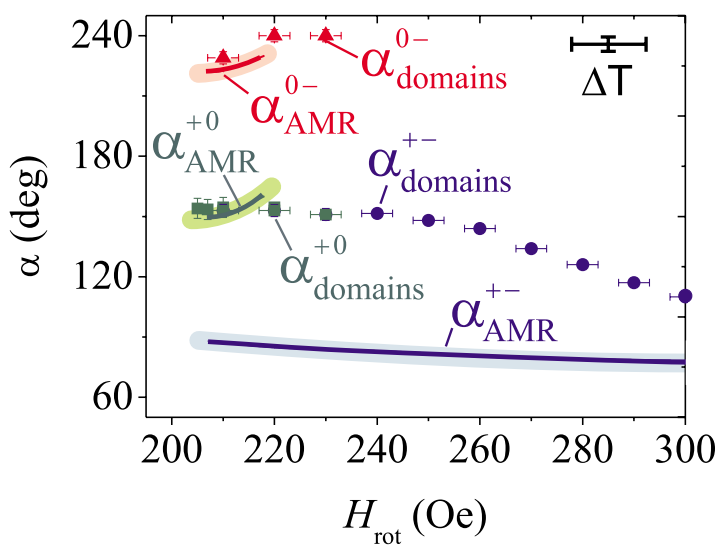

FIG. 5. (Color online) Measured (symbols, "domains") and from the AMR data modeling extracted (lines, "AMR") domain wall orientation angles $\alpha$ as a function of the rotating field magnitude. An intermediate field regime with the $\sigma^{+} \rightarrow \sigma^{0} \rightarrow \sigma^{-}$transition and the beginning of the high field regime with the proposed $\sigma^{+}$ $\rightarrow \sigma^{-}$chirality change can be identified. The additional error due to thermal differences $\Delta \mathrm{T}$ between both experimental setups is indicated.

derived by assuming the magnetic compatibility of the two adjacent domains, i.e., from the requirement of zero net magnetic charge on the LDW, which is fulfilled when the difference between the domain magnetization vectors lies in the wall plane. ${ }^{6}$ In practice, the $1 D$ magnetic profiles at the critical angle of transition were first computed for the two adjacent phases, e.g., $\sigma^{+}$and $\sigma^{0}$. Then, the orientation angle of the LDW, e.g., $\alpha^{+0}$, was determined as the angle such that

$$
\int\left\{\sin \left[\theta^{+}(z)-\alpha^{+0}\right]-\sin \left[\theta^{0}(z)-\alpha^{+0}\right]\right\} d z=0,
$$

where $\theta^{+}(z)$ and $\theta^{0}(z)$ are the spin orientation angles at the depth $z$ in the $\sigma^{+}$state and $\sigma^{0}$ state, respectively.

The results of the calculations are compared with experimental data in Fig. 5. For $H_{\text {rot }}<203$ Oe, the model predicts a single stable state $\sigma^{0}$ and a reversible behavior. Accordingly, no LDW and no transition are observed. In the intermediate field regime (203 Oe $\left.\leq H_{\text {rot }}<220 \mathrm{Oe}\right)$, the two consecutive transitions $\sigma^{+}$to $\sigma^{0}$ and $\sigma^{0}$ to $\sigma^{-}$were clearly observed. Also, the calculated and measured LDW angles $\alpha^{+0}$ and $\alpha^{0-}$ match closely. Lastly, in the high field regime $\left(H_{\mathrm{rot}} \geq 220 \mathrm{Oe}\right)$, where no experimental indication of a twostep reversal was obtained from AMR data, experimental and numerical data do not agree. As already mentioned, the measured LDW orientation angle does not show the strong change expected on the basis of simple arguments. Instead, it follows exactly along the change of $\alpha^{+0}$ with field. From this finding, we derive that the chirality change in the high field regime is still initiated through a $\sigma^{+}$to $\sigma^{0}$ transition. This interpretation is also supported by the $1 D$ model, which proves the existence of a local energy minimum for the $\sigma^{0}$ state, however at field angles below $\psi_{c}$ (around $180^{\circ}$ ).

In the high field regime, the nucleation of the $\sigma^{-}$phase follows immediately that of the $\sigma^{0}$ phase and it takes place at the $\sigma^{+} / \sigma^{0}$ LDWs just formed. This results in the $\sigma^{0}$ phase

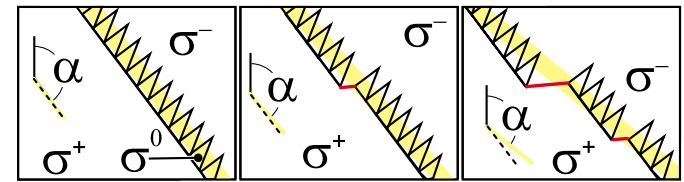

a)

b)

c)

FIG. 6. (Color online) Sketches of the magnetic domain boundaries at modestly high fields [see also inset of Fig. 3(c) for comparison]. The resulting average LDW orientation angles $\alpha$ are indicated and marked along the folded LDWs.

being extremely confined spatially (Fig. 6). Locally, the intermediary $\sigma^{0}$ phase may vanish and a $\sigma^{+} / \sigma^{-}$LDW may form. A hybrid LDW then develops, which consists of $\sigma^{+} / \sigma^{0}$ and $\sigma^{+} / \sigma^{-}$wall segments having two different characteristic orientations. This hybrid structure is at the origin of the irregular shape of the domain boundaries. Evidently, the assumption of just one well-defined type of LDW along the domain boundaries on which we relied to calculate the LDW orientation angles is not valid in this case, hence the discrepancy between calculated and experimental $\alpha^{+-}$data in Fig. 5 . The fact that measured $\alpha_{\text {domains }}^{+-}$approaches the calculated value $\alpha_{\mathrm{AMR}}^{+-}$as the field magnitude increases indicates that the fraction of $\sigma^{+} / \sigma^{-}$wall segments becomes larger, as sketched in Fig. 6. A similar change in the proportions of $\sigma^{+} / \sigma^{0}$ and $\sigma^{+} / \sigma^{-}$wall segments is certainly also at the origin of the small but noticeable variation in LDW alignment with varying field angle [see Figs. 4(c)-4(f)]. In the scenario proposed above, the lateral extent of the intermediary $\sigma^{0}$ phase is beyond the resolution of our magneto-optical microscope.

\section{SUMMARY}

In summary, we combined anisotropic magnetoresistance measurements and direct magnetic domain observations to identify the interfacial domain wall chirality reversal mechanisms in thin-film exchange-spring system. Careful analysis and modeling of orientation of the lateral domain boundaries show that domain nucleation and lateral domain wall propagation are playing the key roles in the chirality reversal of interface planar domain walls in thin-film exchange-spring system. The chirality reversal takes place in two steps. First domains of uniform magnetization nucleate, leading to an unwinding process, then a rewinding occurs via nucleation of inversed chirality domains. The direct chirality reversal between $\sigma^{+}$and $\sigma^{-}$is suppressed and the two phases are found to coexist with the $\sigma^{0}$ phase, forming a network of in-plane and perpendicular domain walls over a large field range. The volume of the intermediate $\sigma^{0}$ state decreases rapidly with magnetic fields, which inhibits a direct detection of the intermediate phase. The results add significant knowledge on the mechanism of magnetization reversal in exchangecoupled systems and will need to be considered to properly describe the magnetization reversal processes in such systems. 
*Stephane.Mangin@1pm.u-nancy.fr

${ }^{1}$ M. Bode, M. Heide, K. von Bergmann, P. Ferriani, S. Heinze, G. Bihlmayer, A. Kubetzka, O. Pietzsch, S. Blügel, and R. Wiesendanger, Nature (London) 447, 190 (2007).

${ }^{2}$ H. A. Dürr, E. Dudzik, S. S. Dhesi, J. B. Goedkoop, G. van der Laan, M. Belakhovsky, C. Mocuta, A. Marty, and Y. Samson, Science 284, 2166 (1999).

${ }^{3}$ R. D. McMichael and M. J. Donahue, IEEE Trans. Magn. 33, 4167 (1997).

${ }^{4}$ J. G. Zhu, Y. F. Zheng, and G. A. Prinz, J. Appl. Phys. 87, 6668 (2000).

${ }^{5}$ S.-B. Choe, Y. Acremann, A. Scholl, A. Bauer, A. Doran, J. Stöhr, and H. A. Padmore, Science 304, 420 (2004).

${ }^{6}$ A. Hubert and R. Schäfer, Magnetic Domains: The Analysis of Magnetic Microstructures (Springer-Verlag, Berlin, 1998).

${ }^{7}$ T. Okuno, K. Shiegeto, T. Ono, K. Mibu, and T. Shinjo, J. Magn. Magn. Mater. 240, 1 (2002).

${ }^{8}$ E. E. Fullerton, J. S. Jiang, and S. D. Bader, J. Magn. Magn. Mater. 200, 392 (1999).

${ }^{9}$ K. V. O’Donovan, J. A. Borchers, C. F. Majkrzak, O. Hellwig, and E. E. Fullerton, Phys. Rev. Lett. 88, 067201 (2002).
${ }^{10}$ M. D. Stiles and R. D. McMichael, Phys. Rev. B 59, 3722 (1999).

${ }^{11}$ A. Scholl, M. Liberati, E. Arenholz, H. Ohldag, and J. Stöhr, Phys. Rev. Lett. 92, 247201 (2004).

${ }^{12}$ V. S. Gornakov, Yu. P. Kabanov, O. A. Tikhomirov, V. I. Nikitenko, S. V. Urazhdin, F. Y. Yang, C. L. Chien, A. J. Shapiro, and R. D. Shull, Phys. Rev. B 73, 184428 (2006).

${ }^{13}$ C. L. Platt, A. E. Berkowitz, S. David, Eric. E. Fullerton, J. S. Jiang, and S. D. Bader, Appl. Phys. Lett. 79, 3992 (2001).

${ }^{14}$ J. S. Jiang, S. D. Bader, H. Kaper, G. K. Leaf, R. D. Shull, A. J. Shapiro, V. S. Gornakov, V. I. Nikitenko, C. L. Platt, A. E. Berkowitz, S. David, and E. E. Fullerton, J. Phys. D 35, 2339 (2002).

${ }^{15}$ F. Montaigne, S. Mangin, and Y. Henry, Phys. Rev. B 67, 144412 (2003).

${ }^{16}$ Y. Henry, S. Mangin, and F. Montaigne, Phys. Rev. B 69, 140401(R) (2004); D. Chumakov, R. Schäfer, D. Elefant, D. Eckert, L. Schultz, S. S. Yan, and J. A. Barnard, ibid. 66, 134409 (2002).

${ }^{17}$ T. Hauet, S. Mangin, J. McCord, F. Montaigne, and E. E. Fullerton, Phys. Rev. B 76, 144423 (2007). 